\title{
Crescimento e fator de condição na fase juvenil de Farfantepenaeus brasiliensis (Latreille) e F. paulensis (Pérez-Farfante) (Crustacea, Decapoda, Penaeidae) em uma lagoa costeira tropical do Rio de Janeiro, Brasil
}

\author{
Edélti Faria Albertoni ${ }^{1,3}$, Cleber Palma-Silva ${ }^{1}$ \& Francisco de Assis Esteves ${ }^{2}$
}

\author{
${ }^{1}$ Laboratório de Ecologia, Departamento de Ciências Morfo-Biológicas, Fundação Universidade Federal do Rio Grande. \\ Campus Carreiros, Avenida Itália Km 8, 96201-900 Rio Grande, Rio Grande do Sul, Brasil. E-mail: dmbefa@super.furg.br \\ ${ }^{2}$ Departamento de Ecologia, Universidade Federal do Rio de Janeiro. Ilha do Fundão, 21941-540 Rio de Janeiro, Rio de \\ Janeiro, Brasil. \\ ${ }^{3}$ Autor correspondente.
}

\begin{abstract}
Growth and condition factor in the juvenile phase of Farfantepenaeus brasiliensis (Latreille) and F. paulensis (Pérez-Farfante) (Crustacea, Decapoda, Penaeidae) in a tropical coastal lagoon of Rio de Janeiro, Brazil. The growth relations of the shrimps Farfantepenaeus brasiliensis (Latreille, 1817) and F. paulensis (Pérez-Farfante, 1967) were evaluated at Imboassica lagoon, Macaé, Rio de Janeiro State, between June 1995 and September 1997, in four periods after events of sandbar opening. This sandbar separated the lagoon from the Atlantic Ocean. Through the evaluation of the weight/length relationships for the two species, significant differences in each interval after the sandbar openings were observed, as much among males and females as among all individuals of the same species. The results indicated that both species presented negative allometric growth in most of the studied occasions. The frequency and abundance of the two species in the lagoon is linked to many factors, among them the season of the sandbar opening, the span of time in which the sandbar remains opened, and the presence of a reproductive stock of adults close to the area when the sandbar is open. Through the analysis of the relative condition factor $(\mathrm{Kr})$, it was verified that usually the conditions inside the lagoon are favorable to the development of both species, with the exception of the occasions when the sandbar opening events happen quickly and close to each other, allowing to conclude that the sandbar openings have an influence on the growth rates and in the general status of the organisms.
\end{abstract}

KEY WORDS. Morphometry, shrimp, weight/length relationship.

Lagoas costeiras em geral são considerados excelentes habitats para crescimento de várias espécies em função sua alta produtividade. A lagoa Imboassica localiza-se na zona urbana do município de Macaé, estado do Rio de Janeiro e, através de eventos de aberturas artificiais da barra de areia que a separa do oceano, muitas espécies de origem marinha penetram na lagoa como larvas e juvenis em busca de condições favoráveis para seu desenvolvimento. Entre estas espécies, os camarões peneídeos Farfantepenaeus brasiliensis (Latreille, 1817) e $F$. paulensis (Pérez-Farfante, 1967) constituem-se em importante recurso para pesca artesanal local.

Os camarões peneídeos constituem-se em um dos recursos pesqueiros mais freqüentes e explorados nas regiões costeiras em todo o mundo, assim como em toda costa brasileira (Mello 1973, Brisson 1977, 1981, Chagas Soares 1985, D'incao 1991, VALENTINI et al. 1991). Farfantepenaeus brasiliensis (Latreille, 1817) distribui-se desde a Carolina do Norte (EUA) até a costa norte do Rio Grande do Sul (Brasil) (29 S) e F. paulensis (Pérez-Farfante, 1967) apresenta uma distribuição mais restrita, desde o cabo de São Tomé (Brasil) $\left(22^{\circ}\right.$ S) até as águas costeiras da província de Buenos Aires, Argentina $\left(38,5^{\circ}\right)$.

A reprodução dos camarões do gênero Farfantepenaeus ocorre na plataforma continental, em profundidades entre 40 e 100 metros; os ovos são bentônicos e, após a eclosão, seguemse três etapas larvais planctônicas, cada uma com vários subestágios (nauplius, protozoea e misis) (BofF \& MARChIORI 1984, D'INCAO 1991), e no estágio pós-larval ocorre a migração das populações para as águas mais calmas e ricas das regiões estuarinas, onde penetram normalmente através das correntes de maré (BRISSON 1981, D’INCAO 1991). Assim, a pesca das espécies de camarão é exercida sobre os dois extratos populacionais em que se distribui cada espécie, ou seja, captura de indivíduos juvenis ou pré-adultos nos criadouros (estuários e lagunas costeiras), e dos adultos no oceano (VAlentini et al. 1991). 
$\mathrm{Na}$ região de Macaé, algumas lagoas costeiras sofrem processos de abertura da barra de areia que as ligam ao oceano. Tais aberturas ocorrem principalmente nas épocas de maior precipitação pluviométrica (meses de verão), quando o nível de água sobe e ocorrem alagamentos nas áreas residenciais próximas. Na lagoa Imboassica, estes eventos provocam grandes mudanças no ecossistema, alterando o metabolismo (FARIA et al. 1998) e as comunidades (Albertoni 1998a, Albertoni et al. 1999, 2001, Branco 1998, Fernandes 1998, Palma-Silva 1998, Palma-Silva et al. 2000). Apesar destas alterações, as aberturas constituem-se na oportunidade de entrada de organismos marinhos, principalmente peixes e crustáceos, para crescimento e desenvolvimento no interior da lagoa (Aguiaro \& CARAMASCHI 1995, Albertoni 1998a, b). Atualmente, a lagoa Imboassica é explorada por poucos pescadores residentes e um número maior de pescadores não-residentes, que atuam quando as condições de pesca são satisfatórias (Frota \& CARAMASCH 1998). Decorridos três a quatro meses dos eventos de abertura de barra, um grande número de pescadores se concentra nas margens da lagoa para a pesca do camarão.

As análises de crescimento das espécies de peneídeos têm sido utilizadas como ferramentas para avaliações de estoques pesqueiros e viabilidade de sobrevivência em locais propícios a cultivos de várias espécies. Estudos de crescimento de várias espécies de peneídeos foram feitos em diversos locais, como os de Lares \& Khandker (1976) na Venezuela; Arreguín-Sánchez (1981) e Álvarez et al. (1987) no México; Subramaniam (1990), em Zanzibar (África); Haywood \& STAples (1993) e Watson \& Turnbull (1993) na Austrália; Palacios et al. (1993), na Costa Rica; Griffith \& Wigglesworth (1993), com espécies do Equador e da Colômbia; no Golfo da Arábia por Mohammed et al. (1996); Castro \& Arreguín-Sánchez (1997), no Golfo do México; e PrimaVERA (1998), nas Filipinas, entre outros.

No Brasil, Farfantepenaeus brasiliensis e F. paulensis são conjuntamente chamados de camarão-rosa e, em geral, em avaliações de estoques pesqueiros a partir de desembarques em entrepostos de pesca, não ocorre diferenciação entre elas (Brisson 1981, Chagas-Soares 1985). Estes estudos têm sido feitos principalmente nas costas sul e sudeste, em locais próximos a grandes regiões de tradição pesqueira, como por exemplo em Santos (estado de São Paulo) (Mello 1973), na região de Cabo Frio (estado do Rio de Janeiro), principalmente na lagoa de Araruama (Brisson 1977, 1981, Villela et al. 1997), e CHAGASSOAREs (1985) em Cananéia (estado de São Paulo). Na região sul, os principais trabalhos com este enfoque foram feitos por BRANCo \& Verani (1998a, b) na lagoa da Conceição (estado de Santa Catarina) e D'InCAO $(1984,1991)$, D'InCAO \& Calazans (1978) e VAlentini et al. (1991) para a região da lagoa dos Patos (estado do Rio Grande do Sul).

O objetivo deste trabalho foi determinar as relações de crescimento das espécies Farfantepenaeus brasiliensis e F. paulensis em sua fase juvenil, na lagoa Imboassica, avaliando as relações peso/comprimento, o fator de condição e as taxas relativas de crescimento, inferindo sobre os efeitos das aberturas da barra da lagoa sobre estas espécies.

\section{MATERIAL E MÉTODOS}

\section{Área de Estudo}

A lagoa Imboassica localiza-se na costa nordeste do estado do Rio de Janeiro, na zona urbana do município de Macaé
( $\left.22^{\circ} 50^{\prime} \mathrm{S} ; 44^{\circ} 42^{\prime} \mathrm{W}\right)$. O clima regional é subúmido, com máxima temperatura média de $29,9^{\circ} \mathrm{C}$ em fevereiro e mínima média em julho $\left(25,4^{\circ} \mathrm{C}\right)$. A precipitação anual varia entre 800 e 1200 mm (RADAmbrasil 1983). Apresenta área de $3,26 \mathrm{Km}^{2}$ e profundidade média de 1,1 m (Panosso et al. 1998) e é separada do oceano por uma barra de areia de aproximadamente $50 \mathrm{~m}$ de largura. Seu entorno é parcialmente ocupado por áreas residenciais onde, algumas vezes, durante a estação chuvosa, ocorrem inundações (BRANCo et al. 1997). Nas épocas de maior pluviosidade são promovidas aberturas artificiais (por ação humana) da barra de areia, não programadas e imprevisíveis, visando sanear os efeitos das inundações nas residências próximas. Estas aberturas causam um drástico abaixamento no nível de água da lagoa, com entrada de água do mar, o que acarreta modificações substanciais nas comunidades bióticas. Quando a lagoa está em comunicação com o oceano, funciona como um estuário, e muitos organismos penetram na mesma para crescimento e alimentação, entre elas os camarões peneídeos Farfantepenaeus brasiliensis and F. paulensis (Albertoni et al. 1999).

\section{Métodos}

Exemplares de Farfantepenaeus brasiliensis e F. paulensis foram coletadas com redes de arrasto e tarrafa, no período de junho/1995 a setembro/1997. Foram realizadas 29 amostragens, em intervalos de aproximadamente 20 dias. As datas das coletas e as variáveis abióticas salinidade (medida com refratômetro), temperatura (medida com termômetro de mercúrio) e profundidade da coluna d'água (obtida com régua graduada) são apresentadas na tabela I. As coletas foram feitas na região próxima à barra da lagoa, à noite, tendo início aproximadamente uma hora após o por do sol. Após a coleta, os organismos foram imediatamente preservados em gelo e levados ao laboratório, para tomada de suas medidas biométricas. As espécies foram identificadas segundo os critérios de Cook (1966), PérezFarfante $(1969,1970,1988)$, Calazans $(1993)$ e Pérez-Farfante \& Kensley (1997).

Foram feitas as medidas de comprimento total $\left(C_{t}\right)$, da extremidade do rostro à extremidade do télson, comprimento da carapaça $\left(\mathrm{C}_{\mathrm{c}}\right)$, da cavidade orbital à extremidade da carapaça, com paquímetro de precisão $0,02 \mathrm{~mm}$, e peso total $\left(\mathrm{P}_{\mathrm{t}}\right)$, em balança digital com precisão de $0,01 \mathrm{~g}$. Os organismos foram pesados após secagem em papel absorvente, para retirada do excesso de água.

A partir destes dados, foram determinadas as relações potenciais de crescimento, para cada espécie, para machos e fêmeas em separado, segundo as equações $\mathbf{P}_{t}=\mathbf{a C}_{t} \mathbf{b}$, e $\mathbf{P}_{t}=\mathbf{a C}_{\mathbf{c}}{ }^{\mathbf{b}}$, onde $\mathrm{P}_{\mathrm{t}}=$ peso total; $\mathrm{C}_{\mathrm{t}}=$ comprimento total; $\mathrm{C}_{\mathrm{c}}=$ comprimento da carapaça; a e b = parâmetros de crescimento (BATSCHELET 1978, Weatherley 1972, Weatherley \& Gill 1987).

Durante o período de estudo ocorreram cinco aberturas artificiais da barra, ocorridas em maio/1995, novembro/1995, abril/1996, novembro/1996 e janeiro/1997, ficando a barra aberta por aproximadamente 14 dias em cada uma delas. $\mathrm{O}$ tempo de recuperação do espelho d'água variou após cada evento, após a abertura de maio/1995 aproximadamente cinco meses, após novembro/1995 três meses, após abril/1996 cinco meses, e após novembro de 1996 menos de 40 dias, com outra abertura sendo realizada já em janeiro de 1997, com aproximadamente três meses para recuperação da profundidade média da lagoa. Desta forma, foram determinados quatro períodos diferenciados para análise do crescimento das populações: 
Tabela I. Datas das coletas e variáveis abióticas medidas na lagoa Imboassica durante o período de estudo, entre junho/1995 e setembro/1997.

\begin{tabular}{|c|c|c|c|}
\hline Data coleta & $\begin{array}{c}\text { Salinidade } \\
(\%)\end{array}$ & $\begin{array}{c}\text { Temperatura } \\
\left({ }^{\circ} \mathrm{C}\right) \\
\end{array}$ & $\begin{array}{c}\text { Profundidade } \\
(\mathrm{cm})\end{array}$ \\
\hline \multicolumn{4}{|c|}{ Abertura 29/V/1995 } \\
\hline $21 / \mathrm{Vl} / 1995$ & 29 & 23,0 & 20 \\
\hline 05/VII/1995 & 15 & 24,0 & 20 \\
\hline 25/VII/1995 & 11 & 21,0 & \\
\hline 11/VIII/1995 & 6 & 19,4 & 40 \\
\hline 24/VIII/1995 & 9 & 22,0 & \\
\hline 22/XI/1995 & 6 & 24,7 & 50 \\
\hline 16/X/1995 & 3 & 24,1 & 90 \\
\hline \multicolumn{4}{|c|}{ Abertura 02/XI/1995 } \\
\hline 13/XI/1995 & 27 & 24,5 & 30 \\
\hline 03/XII/1995 & 14 & 25,7 & 30 \\
\hline 16/XII/1995 & 11 & 29,0 & 60 \\
\hline 27/XII/1995 & 5 & 30,3 & 60 \\
\hline 18/I/1996 & 3 & 30,5 & 80 \\
\hline 26/II/1996 & 1 & 31,0 & 80 \\
\hline 26/III/1996 & 0 & 31,0 & 100 \\
\hline 22/IV/1996 & 0 & 28,0 & 100 \\
\hline \multicolumn{4}{|c|}{ Abertura 26/IV/1996 } \\
\hline 17/VI/1996 & 23 & 24,0 & 20 \\
\hline 01/VII/1996 & 27 & 24,0 & 10 \\
\hline 15/VII/1996 & 18 & 22,5 & 20 \\
\hline 10/VIII/1996 & 9 & 22,0 & 60 \\
\hline 03/IX/1996 & 3 & 24,0 & 85 \\
\hline 27/IX/1996 & 4 & 24,3 & 110 \\
\hline 18/X/1996 & 5 & 23,0 & 130 \\
\hline 05/XI/1996 & 0 & 25,8 & 150 \\
\hline \multicolumn{4}{|c|}{ Abertura16/XI/1996 } \\
\hline 12/XII/1996 & - & - & - \\
\hline \multicolumn{4}{|c|}{ Abertura 06/I/1997 } \\
\hline 05/II/1997 & 15 & 25,7 & 20 \\
\hline 05/III/1997 & 12 & 27,0 & 28 \\
\hline 05/IV/1997 & 8 & 27,0 & 35 \\
\hline 29/IV/1997 & 9 & 23,0 & 50 \\
\hline 15/V/1997 & 0 & 26,0 & 70 \\
\hline 06/VI/1997 & 2 & 20,0 & 97 \\
\hline 03/VII/1997 & 10 & 23,0 & 80 \\
\hline 14/VIII//1997 & 8 & 22,9 & 75 \\
\hline 22/IX/1997 & 6 & 24,7 & 60 \\
\hline
\end{tabular}

a) período 1 - correspondente aos meses de junho/1995 a outubro/1995; b) período 2 - correspondente aos meses de dezembro/1995 a abril/1996; c) período 3 - correspondente aos meses de maio/1996 a novembro/1996; d) período 4 correspondente aos meses de fevereiro/1997 a setembro/1997.

Entre os meses de novembro/1996 e fevereiro/1997 ocorreram duas aberturas de barra (16/XI/1996 e 06/I/1997), sendo por isso os organismos acompanhados somente a partir de fevereiro/1997.

Em cada intervalo entre as aberturas de barra foram obtidas as retas de regressão linear entre comprimento e peso em cada período amostrado, segundo a equação $\ln \mathbf{P}_{t}=\ln \mathbf{a}+\mathbf{b}$ $\operatorname{lnC}_{t^{\prime}}(\ln =$ logaritmo natural) e comparadas, através da análise de covariância (SOKAL \& RoHlF 1979). Para F. paulensis, as análises comparativas foram feitas nos três primeiros intervalos, em função do pequeno número $(\mathrm{n}=5)$ de organismos coletados durante o quarto período. Os coeficientes de crescimento (b) foram comparados com o valor 3 (crescimento isométrico) através do teste " $\mathrm{t}$ " para verificar a tendência de crescimento de machos e fêmeas em cada período.

Foi determinado o Fator de Condição Relativo $\left(\mathrm{K}_{\mathrm{r}}\right)$ para cada espécie, segundo Le Cren (1951), $\mathbf{K}_{\mathrm{r}}=\mathbf{P}_{\text {obs }} / \mathbf{P}_{\text {esp }}$, onde $\mathrm{P}_{\text {esp }}=$ peso esperado, determinado através da curva de pesocomprimento obtida em todo o período, e $\mathrm{P}_{\text {obs }}$ é o peso observado, obtido da pesagem individual de cada organismo. A comparação das médias do fator de condição obtido em cada período foi feita pelo teste $t$ de Student, avaliando a diferença do $\mathrm{K}_{\mathrm{r}}$ médio esperado $(=1) \mathrm{com}$ as médias obtidas, para o total de organismos no período. A análise comparativa do fator de condição relativo entre as duas espécies foi feita através do teste não paramétrico de Mann-Whitney.

A taxa de Crescimento Relativo (G), foi determinada conforme Weatherley (1972) e Weatherley \& Gill (1987), em cada período, para machos e fêmeas separadamente, segundo a equação: $\mathbf{G}=\left(\ln \mathbf{w}_{2}-\ln \mathbf{w}_{1}\right) /\left(\mathbf{t}_{2}-\mathbf{t}_{1}\right)$, onde $\mathrm{w}_{2}$ é o peso médio no instante $t_{2}, w_{1}$ é o peso médio no instante $t_{1}$, e $t_{2}-t_{1} o$ intervalo de tempo entre as duas medidas. Os intervalos de tempo $\left(t_{1}\right.$ e $\left.t_{2}\right)$ considerados para cada espécie e em cada período foram variáveis, e são apresentados os resultados das taxas inicial e final de cada espécie, em cada período considerado. No Período 1, iniciaram-se as coletas com 22 dias decorridos da abertura de barra, no Período 2 com 11 dias, no Período 3 com 42 dias e no Período 4 com 57 dias. Os intervalos de tempo considerados a partir de cada abertura de barra foram: decorridos $22,36,56,71,85$ e 137 dias no primeiro período, $11,31,47,58,80,118,146$ e 175 dias no segundo, 21, 34, 48, $73,96,122$ e 158 dias no período 3 e $57,87,111,127,148,175$ e 254 dias no período 4 .

\section{RESULTADOS}

Foram analisados 1146 indivíduos de F. brasiliensis, 585 fêmeas e 561 machos, e 367 de F. paulensis, 193 fêmeas e 174 machos. A amplitude total de variação no comprimento de $F$. brasiliensis foi de 9 a $145 \mathrm{~mm}$, e para $F$. paulensis foi de 9 a 115 $\mathrm{mm}$. As maiores freqüências ocorreram entre 65 e $100 \mathrm{~mm}$ de comprimento total.

A comparação estatística entre as curvas de $\mathrm{P}_{t} / \mathrm{L}_{\mathrm{t}}$ para $F$. brasiliensis nos quatro períodos de amostragem (Tab. II), mostra que são diferentes entre os períodos e entre machos e fêmeas, exceto entre machos do terceiro e quarto período, e entre 
machos e fêmeas do quarto período. Em virtude da maioria dos resultados apresentar diferença estatística para $\mathrm{p}<0,05$, os dados foram tratados separadamente.

Para F. brasiliensis, as curvas obtidas com a relação $\mathrm{P}_{\mathrm{t}} / \mathrm{L}_{\mathrm{t}^{\prime}}$ em cada intervalo entre aberturas de barra, para machos e fêmeas, e as curvas obtidas entre a relação $P_{t} / L_{c^{\prime}}$ para machos e fêmeas, em cada período (Tab. III), indicam que esta espécie apresentou na maioria das vezes tendência para crescimento alométrico negativo, visualizado pelos coeficientes de crescimento significativamente menores que três $(\mathrm{p}<0,05)$.

Os resultados da comparação estatística entre as curvas obtidas para $F$. paulensis nos três períodos de amostragem são apresentados na tabela IV, apresentando diferença $(\mathrm{p}<0,05)$ entre todos os períodos. As curvas das relações $\mathrm{P}_{t} / \mathrm{L}_{\mathrm{t}}$, e $\mathrm{P}_{\mathrm{t}} / \mathrm{L}_{\mathrm{c}}$ (Tab. V), demonstram que esta espécie apresenta como tendência o crescimento alométrico negativo, verificado pelos coeficientes de crescimento sempre menores $(\mathrm{p}<0,05)$ que três.

Os resultados do fator de condição relativo $\left(\mathrm{K}_{\mathrm{r}}\right)$, comparados com o fator de condição esperado $(=1)$, mostram que $F$. brasiliensis (Tab. VI) apresenta diferentes condições de crescimento conforme o período avaliado. Observa-se que o fator de condição foi significativamente maior de que um, refletindo boas condições de crescimento, nos períodos 1,2 e 3 , exceto para machos do período 2 , onde o $\mathrm{K}_{\mathrm{r}}$ foi igual a um. No quarto período amostral, os $\mathrm{K}_{\mathrm{r}}$ foram significativamente menores que o esperado.

$\mathrm{Na}$ análise dos resultados obtidos para F. paulensis (Tab. VII), observa-se que as condições nos três períodos analisados foram favoráveis ao seu crescimento, refletido pelo $\mathrm{K}_{\mathrm{r}}$ sempre significativamente maior do que um. Como no quarto período a ocorrência desta espécie foi muito baixa $(n=5)$, não foi possível avaliar se as condições encontradas neste período também alterariam suas condição de crescimento relativo. $\mathrm{O}$ $\mathrm{K}_{\mathrm{r}}$ de $F$. paulensis mostrou-se significativamente maior $(\mathrm{p}<$ 0,001 ) do que o de $F$. brasiliensis.

A Taxa de Crescimento Relativo (G) calculada em cada período, para as duas espécies, demonstra que, em todos os períodos, o crescimento atinge taxas elevadas nas primeiras fases do ciclo vital, diminuindo para um ritmo mais lento com o decorrer do tempo. Para F. paulensis, não foi possível o cálculo desta taxa nos períodos 2 e 4, em função do baixo $n$ amostral.

As estimativas do crescimento relativo das duas espécies, em $\mathrm{g} \mathrm{d}^{-1}$, mostram variações principalmente em seus valores iniciais, em função do tempo decorrido entre a abertura de barra e os primeiros tamanhos de organismos capturados, e variações entre machos e fêmeas (Tabs VIII e IX).

Tabela II. Matriz do teste de comparação (ANCOVA, $\mathrm{p}<0,05$ ) entre as curvas de peso-comprimento total obtidas para Farfantepenaeus brasiliensis nos quatro períodos avaliados, entre junho/95 e setembro/97, na lagoa Imboassica. $\left({ }^{*}\right)$ Valores não-significativos.

\begin{tabular}{|c|c|c|c|c|c|c|c|c|}
\hline \multirow{2}{*}{ Período } & \multicolumn{2}{|c|}{1} & \multicolumn{2}{|c|}{2} & \multicolumn{2}{|c|}{3} & \multicolumn{2}{|c|}{4} \\
\hline & Machos & Fêmeas & Machos & Fêmeas & Machos & Fêmeas & Machos & Fêmeas \\
\hline \multirow{2}{*}{1} & Machos & & & & & & & \\
\hline & Fêmeas & 0,000032 & & & & & & \\
\hline \multirow{2}{*}{2} & Machos & 0,000032 & 0,000032 & & & & & \\
\hline & Fêmeas & 0,000032 & 0,000032 & 0,000032 & & & & \\
\hline \multirow{2}{*}{3} & Machos & 0,000032 & 0,000032 & 0,018783 & 0,000032 & & & \\
\hline & Fêmeas & 0,000032 & 0,000032 & 0,000032 & 0,000032 & 0,000032 & & \\
\hline \multirow{2}{*}{4} & Machos & 0,000032 & 0,000032 & 0,002964 & 0,000032 & $0,996079 *$ & 0,000032 & \\
\hline & Fêmeas & 0,000032 & 0,000032 & 0,000032 & 0,000032 & 0,015539 & 0,000032 & $0,157643^{*}$ \\
\hline
\end{tabular}

Tabela III. Relação de Peso Total $\times$ Comprimento Total $\left(\mathrm{P}_{\mathrm{t}} \times \mathrm{C}_{\mathrm{t}}\right)$ e Peso Total $\times$ Comprimento da Carapaça $\left(\mathrm{P}_{\mathrm{t}} \times \mathrm{C}_{\mathrm{c}}\right)$ de Farfantepenaeus brasiliensis em cada período de intervalo entre aberturas de barra. ( $\left.{ }^{*}\right)$ Valores não-significativos para $b \neq 3$.

\begin{tabular}{|c|c|c|c|c|c|c|}
\hline Período & & $P_{t} \times C_{t}$ & $r^{2}$ & $P_{t} \times C_{c}$ & $r^{2}$ & $\mathrm{n}$ \\
\hline \multirow{2}{*}{1} & Machos & $P_{t}=0,00001 C_{t}^{2,9062 *}$ & 0,99 & $P_{t}=0,0003 C_{c}^{2,8635 *}$ & 0,98 & 62 \\
\hline & Fêmeas & $P_{t}=0,00001 C_{t}^{2,9155 *}$ & 0,99 & $P_{t}=0,0002 C_{c}^{3,0319}$ & 0,99 & 61 \\
\hline \multirow{2}{*}{2} & Machos & $P_{t}=0,00001 C_{t}^{2,8837 *}$ & 0,98 & $P_{t}=0,0004 C_{c}^{3,1282 *}$ & 0,73 & 85 \\
\hline & Fêmeas & $P_{t}=0,00003 C_{t}^{2,6928 *}$ & 0,97 & $P_{t}=0,0001 C_{c}^{3,5285 *}$ & 0,62 & 92 \\
\hline \multirow{2}{*}{3} & Machos & $P_{t}=0,00002 C_{t}^{2,8349 *}$ & 0,98 & $P_{t}=0,0008 C_{c}^{2,9868}$ & 0,95 & 236 \\
\hline & Fêmeas & $P_{t}=0,000009 C_{t}^{2,9596 *}$ & 0,98 & $P_{t}=0,001 C_{c}^{2,9069 *}$ & 0,96 & 247 \\
\hline \multirow{2}{*}{4} & Machos & $P_{t}=0,000006 C_{t}^{3,0334 *}$ & 0,98 & $P_{t}=0,0004 C_{c}^{3,2206 *}$ & 0,97 & 178 \\
\hline & Fêmeas & $P_{t}=0,000005 C_{t}^{3,0754 *}$ & 0,99 & $P_{t}=0,0004 C_{c}^{3,1722 *}$ & 0,98 & 185 \\
\hline
\end{tabular}

Revista Brasileira de Zoologia 20 (3): 409-418, setembro 2003 
Tabela IV. Matriz do teste de comparação (ANCOVA, $p<0,05$ ) entre as curvas de peso-comprimento total obtidas para Farfantepenaeus paulensis nos três períodos avaliados, entre junho/1995 e novembro/1996, na lagoa Imboassica. Todos os valores significativos.

\begin{tabular}{ccccccc}
\hline Período & \multicolumn{2}{c}{1} & \multicolumn{2}{c}{2} & \\
\hline & Machos & Fêmeas & Machos & Fêmeas & Machos & Fêmeas \\
1 & Machos & & & & & \\
& Fêmeas & 0,000020 & & & & \\
2 & Machos & 0,000020 & 0,000020 & & & \\
& Fêmeas & 0,000868 & 0,000020 & 0,007808 & & \\
& Machos & 0,000020 & 0,000020 & 0,000020 & 0,000020 & \\
& Fêmeas & 0,000020 & 0,000020 & 0,000020 & 0,000020 & 0,000020 \\
\hline
\end{tabular}

Tabela V. Relações de Peso Total $\times$ Comprimento Total $\left(P_{t} \times C_{t}\right)$ e Peso Total $\times$ Comprimento da Carapaça $\left(P_{t} \times C_{t}\right)$ de Farfantepenaeus paulensis em cada período de intervalo entre aberturas de barra. Todos valores significativos para $b \neq 3$.

\begin{tabular}{|c|c|c|c|c|c|c|}
\hline Período & & $P_{t} \times C_{t}$ & $r^{2}$ & $P_{t} \times C_{c}$ & $\mathrm{r}^{2}$ & $\mathrm{n}$ \\
\hline \multirow{2}{*}{1} & Machos & $\mathrm{P}_{\mathrm{t}}=0,00001 \mathrm{C}_{\mathrm{t}}^{2,8906 *}$ & 0,99 & $P_{t}=0,0003 C_{c}^{2,8476 *}$ & 0,99 & 118 \\
\hline & Fêmeas & $P_{t}=0,00001 C_{t}^{2,9169 *}$ & 0,99 & $\mathrm{P}_{\mathrm{t}}=0,0002 \mathrm{C}_{\mathrm{c}}^{2,9815 *}$ & 0,99 & 129 \\
\hline \multirow{2}{*}{2} & Machos & $P_{t}=0,00001 C_{t}^{2,8497 *}$ & 0,99 & $P_{t}=0,0004 C_{c}^{2,8542 *}$ & 0,95 & 16 \\
\hline & Fêmeas & $P_{t}=0,00003 C_{t}^{2,8405 *}$ & 0,96 & $\mathrm{P}_{\mathrm{t}}=0,0001 \mathrm{C}_{\mathrm{c}}^{2,8026 *}$ & 0,66 & 13 \\
\hline \multirow{2}{*}{3} & Machos & $\mathrm{P}_{\mathrm{t}}=0,00002 \mathrm{C}_{\mathrm{t}}^{2,7329 *}$ & 0,98 & $P_{t}=0,0008 C_{c}^{2,9739 *}$ & 0,94 & 40 \\
\hline & Fêmeas & $\mathrm{P}_{\mathrm{t}}=0,000009 \mathrm{C}_{\mathrm{t}}^{2,9257 *}$ & 0,99 & $\mathrm{P}_{\mathrm{t}}=0,001 \mathrm{C}_{\mathrm{c}}^{2,8402 *}$ & 0,95 & 51 \\
\hline
\end{tabular}

Tabela VI. Resultados do teste $t$ de Student na comparação de médias dos fatores de condição relativo $\left(\mathrm{K}_{\mathrm{r}}\right)$ obtidos para Farfantepenaeus brasiliensis, para machos e fêmeas em cada período de intervalo entre aberturas de barra. (*) Significância estatística com $p<0,05$, (n) número de organismos no período, (SD) desvio padrão da média.

\begin{tabular}{|c|c|c|c|c|c|}
\hline Sexo & $\begin{array}{c}\mathrm{K}_{\mathrm{r}} \\
\text { esperado }\end{array}$ & $\begin{array}{l}\mathrm{K}_{\mathrm{r}} \text { médio } \\
\text { observado }\end{array}$ & $\mathrm{N}$ & SD & $\mathrm{T}_{\text {calc }}$ \\
\hline Fêmeas 1 & 1 & 1,2095 & 61 & 0,158 & $10,34^{*}$ \\
\hline Machos 1 & 1 & 1,1619 & 62 & 0,088 & $14,48^{*}$ \\
\hline Fêmeas 2 & 1 & 1,1479 & 92 & 0,493 & $2,89^{*}$ \\
\hline Machos 2 & 1 & 0,9925 & 85 & 0,303 & 0,22 \\
\hline Fêmeas 3 & 1 & 1,0260 & 247 & 0,058 & $7,04^{*}$ \\
\hline Machos 3 & 1 & 1,0231 & 236 & 0,053 & 6,67 \\
\hline Fêmeas 4 & 1 & 0,9412 & 185 & 0,051 & 15,62 \\
\hline Machos 4 & 1 & 0,9381 & 178 & 0,057 & $14,52^{*}$ \\
\hline
\end{tabular}

\section{DISCUSSÃO}

As relações entre o peso e comprimento de uma espécie são importantes na determinação de sua condição de sobrevivência em um determinado habitat, provendo as bases para estimativas de crescimento e produção, e descrevendo as
Tabela VII. Resultados do teste t de Student na comparação de médias dos fatores de condição relativo $\left(K_{r}\right)$ obtidos para Farfantepenaeus paulensis, para machos e fêmeas em cada período de intervalo entre aberturas de barra. (*) Significância estatística com $\mathrm{p}<0,05$, (n) número de organismos no período, (SD) desvio padrão da média.

\begin{tabular}{ccccccc}
\hline Sexo & & $\begin{array}{c}\mathrm{K}_{\mathrm{r}} \\
\text { esperado }\end{array}$ & $\begin{array}{c}\mathrm{K}_{\mathrm{r}} \text { médio } \\
\text { observado }\end{array}$ & $\mathrm{N}$ & $\mathrm{SD}$ & $\mathrm{T}_{\text {calc }}$ \\
\hline Fêmeas & 1 & 1 & 1,179 & 129 & 0,063 & 32,28 * \\
Machos & 1 & 1 & 1,127 & 118 & 0,075 & 18,30 * \\
Fêmeas & 2 & 1 & 1,460 & 13 & 0,064 & 26,67 * \\
Machos & 2 & 1 & 1,467 & 16 & 0,059 & 32,46 * \\
Fêmeas & 3 & 1 & 1,453 & 51 & 0,073 & 44,48 * \\
Machos & 3 & 1 & 1,439 & 40 & 0,089 & 31,57 * \\
\hline
\end{tabular}

características estruturais dos indivíduos nas populações (ANDERSON \& Gutreuter 1989). As taxas de crescimento de muitos organismos variam por razões intrínsecas (efeitos genéticos) e extrínsecas (variação de disponibilidade de alimento, temperatura, etc) (Alford \& Jackson 1993). Segundo Kauffman (1981), a variação do tamanho e do peso dos organismos, ao longo de um determinado intervalo de tempo, sugerem que estes fatores determinam as taxas de crescimento de uma espécie.

Revista Brasileira de Zoologia 20 (3): 409-418, setembro 2003 
Tabela VIII. Valores inicial e final da Taxa Relativa de Crescimento $(\mathrm{G})\left(\mathrm{gd}^{-1}\right)$,para Farfantepenaeus brasiliensis, nos quatro períodos entre aberturas de barra, na lagoa Imboassica, entre junho/1995 a setembro/1997. O intervalo de dias mostra o tempo decorrido de cada abertura.

\begin{tabular}{ccccc}
\hline Períodos & $\mathrm{G}$ inicial machos $\left(\mathrm{g} \mathrm{d}^{-1}\right)$ & $\mathrm{G}$ final machos $\left(\mathrm{g} \mathrm{d}^{-1}\right)$ & $\mathrm{G}$ inicial fêmeas $\left(\mathrm{g} \mathrm{d}^{-1}\right)$ & $\mathrm{G}$ final fêmeas $\left(\mathrm{g} \mathrm{d}^{-1}\right)$ \\
\hline Período 1 (22 a 137 dias) & 0,221 & 0,0180 & 0,209 & 0,015 \\
Período 2 (11 a 175 dias) & 0,236 & 0,0070 & 0,193 & 0,009 \\
Período 3 (42 a 158 dias) & 0,017 & 0,0070 & 0,046 & 0,006 \\
Período 4 (57 a 254 dias) & 0,016 & 0,0008 & 0,024 & 0,001 \\
\hline
\end{tabular}

Tabela IX. Valores inicial e final da Taxa Relativa de Crescimento $(\mathrm{G})\left(\mathrm{gd}^{-1}\right)$,para Farfantepenaeus paulensis, em dois períodos entre aberturas de barra, na lagoa Imboassica, entre junho/1995 a setembro/1997. O intervalo de dias mostra o tempo decorrido de cada abertura.

\begin{tabular}{lcccc}
\hline & $\mathrm{G}$ inicial machos $\left(\mathrm{g} \mathrm{d}^{-1}\right)$ & $\mathrm{G}$ final machos $\left(\mathrm{g} \mathrm{d}^{-1}\right)$ & $\mathrm{G}$ inicial fêmeas $\left(\mathrm{g} \mathrm{d}^{-1}\right)$ & $\mathrm{G} \mathrm{final} \mathrm{fêmeas}\left(\mathrm{g} \mathrm{d}^{-1}\right)$ \\
\hline Período 1 (22 a 137 dias) & 0,325 & 0,008 & 0,334 & 0,019 \\
Período 3 (42 a 158 dias) & 0,025 & 0,002 & 0,035 & 0,009 \\
\hline
\end{tabular}

Mudanças nas condições ambientais nas áreas de crescimento de peixes e crustáceos juvenis podem afetar o tamanho dos adultos de uma população (Staples \& Heales 1991). As populações de camarão da lagoa Imboassica estão sujeitas a uma gama de fatores ambientais, que vão além de simples variações em temperatura e salinidade, mas também de como estes fatores atuam sobre a estrutura das comunidades que possam ser importantes recursos para os camarões.

O processo de abertura artificial da barra de areia da lagoa Imboassica traz conseqüências a todo o metabolismo do ecossistema (Esteves 1998, Faria et al. 1998), afetando as comunidades bióticas de diferentes maneiras. A salinidade da lagoa, em períodos que antecedem as aberturas, varia de $1 \mathrm{a} 0 \mathrm{ppm}$, e logo após o evento, na região próxima à barra, atinge valores equivalentes à água marinha, na casa de $33 \%$, diminuindo, gradualmente, a medida que o corpo da lagoa vai sendo preenchido com água doce proveniente do rio Imboassica e de precipitação pluviométrica (Petrucio 1998). No período em que permanece com a barra aberta, a lagoa Imboassica funciona como um sistema estuarino aberto, sujeita ao regime de correntes de maré (Frota \& Caramaschi 1998). Desta forma, o sistema adquire inicialmente características mesohalinas, que passam a oligohalinas no decorrer do tempo, com as regiões mais interiores caracteristicamente dulcícolas (BRANCO 1998).

Como a lagoa Imboassica não possui ligação permanente com o mar, a entrada de espécies marinhas de camarão é dependente de vários fatores, que devem combinar a época de pico reprodutivo do estoque adulto no oceano, a abertura de barra, o estoque de pós-larvas nas proximidades da costa, e as correntes oceânicas predominantes, que em última instância, são o meio pelo qual as pós-larvas se deslocam. Desta forma, a ocorrência e abundância de larvas de origem marinha na lagoa pode variar muito a cada evento de abertura (AlBERTONi et al. 1999).

No período deste estudo, entre junho de 1995 e setembro de 1997, foram registrados 5 eventos de abertura de barra, em maio e novembro de 1995, em abril e novembro de 1996, e em janeiro de 1997. A recuperação do corpo lagunar apresentou características distintas em cada uma delas, observando-se que o tempo de recuperação do espelho d'água variou desde aproximadamente cinco meses (após maio de 1995), até pouco menos de 40 dias, como a abertura de novembro de 1996, seguida de outra no início de janeiro de 1997.

Esta variabilidade se refletiu na abundância de pós-larvas de peneídeos e paleomonídeos (AlBeRTONi et al. 1999) e na própria composição das espécies que penetram na lagoa. Assim, no primeiro período avaliado, $F$. paulensis foi capturada em números superiores à $F$. brasiliensis, fato que se inverteu no decorrer dos outros períodos, com baixa abundância de $F$. paulensis principalmente no segundo e quarto períodos. Isto provavelmente se deve a relações com a disponibilidade de larvas no oceano,que se reflete em diferentes épocas de recrutamento. Variações na composição de espécies de peixes a cada evento de abertura foram relatadas por FROTA \& CARAMASCHI (1998).

A cada período após as aberturas, o estoque de camarões é renovado, sendo capturados somente organismos jovens e que seguem um ciclo de crescimento. Durante os períodos de intervalos entre as aberturas, decorridos aproximadamente 4 meses, o estoque populacional diminui rapidamente, pois intensifica-se a pesca artesanal destes crustáceos na lagoa. Nas amostragens realizadas, este fato é detectado pela baixa freqüência das classes de tamanho maiores que $100 \mathrm{~mm}$. Desta forma, se não ocorrer uma nova abertura, a tendência é de que o estoque diminua abruptamente, pois as espécies não se reproduzem no interior da lagoa.

As relações de peso/comprimento obtidas para as duas espécies são semelhantes às reportadas na literatura. Segundo Mello (1973), as relações de Penaeus brasiliensis (= Farfantepenaeus brasiliensis), a partir de amostras desembarcadas no porto de Santos (São Paulo), são diferenciadas para machos $\left(\mathrm{P}_{\mathrm{t}}=\right.$ $\left.0,0062 C_{t}^{3,11}\right)$ e fêmeas $\left(P_{t}=0,0101 C_{t}^{2,96}\right)$. No México, ArreguínSANCHEZ (1981) chegou às relações de $P_{t}=0,00002493 C_{t}^{2,2718}$ para machos e $P_{t}=0,00001005 C_{t}^{2,9677}$ para fêmeas, também com amostras desembarcadas em entrepostos de pesca. Trabalhando com indivíduos juvenis e pré-adultos da espécie na lagoa da Conceição (estado de Santa Catarina), Branco \& VERANi (1998a), obtiveram as seguintes relações: machos $P_{t}=0,0066 C_{t}^{3,0812}$, e fêmeas $P_{t}=0,0100 C_{t}^{2,8916}$. Na lagoa de Araruama (estado do Rio de Janeiro), Villela et al. (1997) encontraram as expressões $\mathrm{P}=0,0012 \mathrm{C}_{\mathrm{t}}^{2,73}$ para as fêmeas, e $\mathrm{P}=0,0014 \mathrm{C}_{\mathrm{t}}^{2,61}$ para os machos. 
Os resultados obtidos para esta espécie, pelos autores acima, indicam que a mesma apresenta crescimento alométrico negativo na maioria dos casos analisados, corroborando os dados obtidos no presente trabalho, onde a maioria dos coeficientes de crescimento foram menores que três, com exceção do quarto período, o que indica esta tendência. $\mathrm{O}$ valor três representa crescimento isométrico, ou seja, o animal cresce simetricamente; valores menores representam crescimento alométrico negativo (animal aumenta mais em comprimento do que em peso) e maiores que três, crescimento alométrico positivo, onde o animal aumenta proporcionalmente mais em peso do que em comprimento (TESH 1968).

De forma semelhante, $F$. paulensis apresentou coeficientes de crescimento sempre menores que 3 , resultado que corrobora os de Mello (1973), que obteve para machos a expressão $P_{t}=$ $0,0106 C_{t}^{2,87}$ e para fêmeas $P_{t}=0,0108 C_{t}^{2,90}$. Na lagoa da Conceição (estado de Santa Catarina), Branco \& Verani (1998b) obtiveram as seguintes equações: machos $P_{t}=0,0098 C_{t}^{3,8853}$, e fêmeas $P_{t}=0,0172 C_{t}^{2,6140}$.

As relações de peso total com comprimento da carapaça demonstraram a mesma tendência para Farfantepenaeus paulensis, corroborando os resultados de ZENKNER \& AgNes (1977), que determinaram coeficientes de 2,79 para machos e 2,69 para fêmeas. D'Incao \& Calazans (1978), por outro lado, demonstraram que na lagoa dos Patos (estado do Rio Grande do Sul), estes coeficientes atingem 3,17 para os machos e 3,04 para as fêmeas, concluindo que o ganho em peso, nesta lagoa, é maior do que em outras áreas analisadas.

Farfantepenaeus brasiliensis apresentou, durante os períodos analisados, diferenças entre os coeficientes de crescimento. Assim, no período 1, que correspondeu aos meses de junho a outubro de 1995, e no período 3 , correspondente aos meses de maio a novembro de 1996 , os coeficientes de crescimento determinados estiveram abaixo do valor 3 , e nos períodos 2 (entre dezembro de 1995 e abril de 1996) e 4 (entre fevereiro de 1997 e setembro de 1997), estes valores foram superiores. Estas diferenças podem refletir as diferentes condições ambientais da lagoa que, como já mencionado, podem sofrer alterações em função das diferentes épocas de aberturas, pelo período em que a barra fica aberta, e a velocidade de recuperação do corpo de água.

As análises de dados de peso/comprimento individualmente em uma espécie fornecem indicações das condições gerais de um organismo em relação ao ambiente, e estágios de desenvolvimento, através da estimativa do fator de condição relativo ( $\mathrm{K}_{\mathrm{r}}$ ) (Le CREN 1951). Segundo o autor, este fator indica o grau de bem estar do organismo frente ao meio em que vive, fornecendo indicações quando se deseja comparar duas ou mais populações vivendo em determinadas condições ambientais, e acompanhar o grau de atividade alimentar de uma espécie, verificando se ela está ou não fazendo bom uso de seus recursos alimentares (WEATHERLEY 1972, WeATHERLEY \& Gill 1987). Alguns autores o tem utilizado em populações de peixes (VAzzoler \& Vazzoler 1965, Braga 1986, 1997, entre outros), e crustáceos (Du Preez \& McLachlan 1984).

Como as curvas da relação peso/comprimento foram diferentes estatisticamente em cada período analisado, e entre machos e fêmeas (Tabs I e II), o fator de condição relativo foi estimado também diferencialmente. Os diferentes resultados permitem supor variações nas condições do meio, que se refletiram no crescimento das espécies de camarão. Assim, as condições nos três primeiros períodos analisados mostraramse favoráveis para o crescimento das duas espécies, que atingiram $\mathrm{K}_{\mathrm{r}}$ maiores que um.

Farfantepenaeus brasiliensis apresentou o fator de condição relativo menor que 1 no quarto período avaliado, indicando que as condições de crescimento foram diferentes nesta época. A espécie $F$. paulensis não foi avaliada neste período, em função de sua baixa freqüência amostral. Provavelmente o efeito provocado por duas aberturas artificiais consecutivas de barra, em um intervalo de aproximadamente 40 dias (em meados de novembro de 1996 e no início de janeiro de 1997), tenha provocado sérios danos ao ecossistema, prejudicando o estabelecimento e a recuperação das comunidades na lagoa, entre elas a comunidade bentônica e a fauna associada a macrófitas aquáticas (AlBERTONi et al. 2001), que constituem o principal recurso alimentar para estas espécies (AlBerToNi et al. 2003 e no prelo). Em uma comparação geral de todo o período estudado, com o $\mathrm{K}_{\mathrm{r}}$ médio de todos os organismos, os resultados mostraram que $F$. paulensis, $\left(\mathrm{K}_{\mathrm{r} \text { méd }}=1,1562\right)$, atinge melhores condições de crescimento na lagoa Imboassica que F. brasiliensis, $\left(\mathrm{K}_{\mathrm{r} \text { méd }}=1,0158\right)$ mesmo que para as duas espécies o fator de condição mostrou-se estatisticamente maior do que um.

As taxas de crescimento são empregadas, freqüentemente, como um dos métodos mais úteis de apresentação de dados de crescimento, permitindo ao investigador fazer comparações de períodos relativamente curtos de crescimento, seja entre populações diferentes, ou entre vários períodos do ciclo vital de uma determinada população (CHAPMAN 1968, WeATHERLeY 1972, WeATHERLEY \& Gill 1987). Estas taxas normalmente decaem ao longo do ciclo vital do animal, sendo elevadas nos primeiros estágios, diminuindo até atingirem valores baixos conforme o organismo se aproxima de sua idade máxima.

Para as espécies avaliadas, as curvas resultantes do cálculo das taxas de crescimento relativo (G), demonstram a tendência de diminuição ao longo do ciclo de crescimento das espécies, com velocidades maiores nas primeiras etapas da vida, e próximo de zero quando nos comprimentos de pré-adultos, momento em que, nos sistemas estuarinos abertos, iniciariam a migração para reprodução no oceano. Os resultados mostram que as taxas iniciais de crescimento foram elevadas no período 1 para ambas as espécies, e também no período 2 para $F$. brasiliensis. Nos outros períodos, os valores são um pouco menores no início da avaliação, mas mantendo a tendência de diminuição ao longo do ciclo vital.

Através dos resultados de crescimento de F. brasiliensis e F. paulensis na lagoa Imboassica, pode-se considerar que esta lagoa, mesmo sujeita a impactos de origem antrópica, atua como um local favorável ao crescimento das duas espécies. Deve ser dada atenção, no entanto, a um manejo e planejamento dos processos de abertura de barra que, embora possibilitem a entrada destas espécies na lagoa, podem afetar de modo abrupto o ecossistema, trazendo sérios danos às comunidades bióticas do local. Isto fica evidenciado quando, após um período de duas aberturas consecutivas em um prazo muito curto, as condições de crescimento mostraram-se menos favoráveis.

\section{CONCLUSÕES}

As espécies Farfantepenaeus brasiliensis e Farfantepenaeus paulensis encontram condições favoráveis na lagoa Imboassica, apresentando crescimento alométrico negativo. As condições

Revista Brasileira de Zoologia 20 (3): 409-418, setembro 2003 
resultantes após cada evento de abertura artificial de barra influenciam nas taxas de crescimento e na condição geral dos organismos na lagoa. Aberturas muito freqüentes causam efeito negativo sobre o crescimento das espécies, como verificado pelos valores do Fator de Condição Relativo, para Farfantepenaeus brasiliensis, no quarto período, após duas aberturas sucessivas em um curto intervalo de tempo.

\section{AGRADECIMENTOS}

Os autores agradecem ao CNPq, CAPES e PETROBRAS pelo suporte financeiro.

\section{REFERÊNCIAS BIBLIOGRÁFICAS}

Aguiaro, T. \& E.P. Caramaschi. 1995. Icthyofauna composition of three coastal lagoons in the north of the State of Rio de Janeiro (Brazil). Brazilian Archives of Biology and Tecnology, Curitiba, 38: 1181-1189

Albertoni, E.F. 1998a. Camarões da lagoa Imboassica: dados preliminares e perspectivas de manejo, p. 359-371. In: F.A. Esteves (Ed.) Ecologia das Lagoas Costeiras do Parque Nacional da Restinga de Jurubatiba e do Município de Macaé (RJ). Rio de Janeiro, NUPEM/UFRJ, 464p.

1998b. Ocorrência de camarões Peneídeos e Paleomonídeos nas lagoas Imboassica, Cabiúnas, Comprida e Carapebus, p. 351-358. In: F.A. Esteves (Ed.) Ecologia das Lagoas Costeiras do Parque Nacional da Restinga de Jurubatiba e do Município de Macaé (RJ). Rio de Janeiro, NUPEM/UFRJ, 464p.

Albertoni, E.F.; C. Palma-Silva; F.A. Esteves. 1999. Larvae and postlarvae of Penaeidae and Palaemonidae in costal lagoons of the north of Rio de Janeiro (Macaé, RJ). Revista Brasileira de Biologia, Rio de Janeiro, 59 (1): 109-117.

. 2001. Macroinvertebrates associated with Chara in a tropical coastal lagoon (Brazil). Hydrobiologia, Dordrecht, 457 (1/3): 215-224.

. 2003. Overlap of Dietary Niche and Electivity of Three Shrimp Species (Crustacea: Decapoda) in a Tropical Coastal Lagoon (Rio de Janeiro, Brazil). Revista Brasileira de Zoologia, Curitiba, 20 (1): 135-140.

- (no prelo). Natural diet of three species of shrimp in a tropical coastal lagoon (Imboassica lagoon, RJ, Brazil). Brazilian Archives of Biology and Tecnology, Curitiba.

Alford, R. A. \& G.D. Jackson. 1993. Do cephalopods and larvae of other taxa grew asymptotically? The American Naturalist, Chicago, 141 (5): 717-728.

Álvarez, N. F.; G.A Gracia \& L.A Soto. 1987. Crecimento y mortalidad de las fases estuarinas del camarón rosado Penaeus (Farfantepenaeus) duorarum Burkenroad, 1939 en la laguna de Términos, Campeche, México. Annales del Instituto de Ciencias del Mar y Limnología de la Universidad National Autónoma de México, México, 14 (2): 207-220.

Anderson, R. \& S. Gutreuter. 1989. Lenght, weigth and associated structural indices, p. 283-300. In: L. Nielsen \& D. JoHNSON (Eds) Fisheries Techniques. Columbus, American Fisheries Society Publications, 468p.

Arreguín-Sánchez, F. 1981. Tasa de crecimiento del camaron rojo (Penaeus brasiliensis Latreille, 1817) de las costas de Quintana Roo, Mexico. Ciencia Pesquera, México 1 (1): 61-70.
BATSCHeLET, E. 1978. Introdução à matemática para Biocientistas. São Paulo, Interciência/EDUSP, 596p.

BofF, M.H. \& M.A. Marchiori. 1984. The effect of temperature on larval development on the pink shrimp Penaeus paulensis. Atlântica, Rio Grande, 7: 7-13.

BRAGA, F.M.S. 1986. Estudo entre fator de condição e relação peso/comprimento para alguns peixes marinhos. Revista Brasileira de Biologia, Rio de Janeiro, 46 (2): 339-346.

. 1997. Análise da equação alométrica na relação peso e comprimento e o fator de condição em Plagioscion squamosissimus (Teleostei, Sciaenidae). Revista Brasileira de Biologia, Rio de Janeiro 57 (3): 417-425.

Branco, C.W.C. 1998. Composição e aspectos ecológicos das comunidades zooplanctônicas nas lagoas Imboassica, Cabiúnas e Comprida, p. 247-271. In: F.A. Esteves.(Ed.). Ecologia das Lagoas Costeiras do Parque Nacional da Restinga de Jurubatiba e do Município de Macaé (RJ). Rio de Janeiro, NUPEM/UFRJ, 464p.

Branco, C.W.C.; T. Aguiaro; F.A. Esteves \& E.P. Caramaschi. 1997. Food sources of the teleost Eucinostomus argenteus in two costal lagoons of Brazil. Studies in Neotropical Fauna \& Environment, Lisse, 32: 33-40.

Branco, J.O. \& J.R. Verani. 1998a. Aspectos bioecológicos do camarão-rosa Penaeus brasiliensis Latreille (Natantia, Penaeidae) da lagoa da Conceição, Florianópolis, Santa Catarina, Brasil. Revista Brasileira de Zoologia, Curitiba, 15 (2): $345-351$.

—. 1998b. Estudo populacional do camarão-rosa Penaeus paulensis Pérez-Farfante (Natantia, Penaeidae) na lagoa da Conceição Santa Catarina, Brasil. Revista Brasileira de Zoologia, Curitiba, 15 (2): 353-364.

BRIsson, S. 1977. Estudo da população de peneídeos na área de Cabo Frio. II - Distribuição sazonal de post-larvas de camarão "rosa" (Penaeus brasiliensis Latreille e Penaeus paulensis Pèrez-Farfante) na entrada do canal da laguna de Araruama - Cabo Frio - Rio de Janeiro, Brasil. Publicações do Instituto Pesqueiro da Marinha, Rio de Janeiro, 101: 1-19.

BRISSON, S. 1981. Estudo da população de peneídeos da área de Cabo Frio. IV - Limite da penetração das post-larvas de camarão-rosa na Laguna de Araruama. Publicações do Instituto Pesqueiro da Marinha, Rio de Janeiro, 141: 1-11.

Calazans, D. 1993. Key to the larvae and decapodits of genera of the infraorder Penaeidea from the southern Brazilian coast. Nauplius, Rio Grande, 1: 45-62.

Castro, R.G. \& F. Arreguín-Sànchez 1997. Mortality and stock assessment of the brown Shrimp, Penaeus aztecus (Crustacea: Penaeidae), in the northwest Gulf of Mexico. Revista de Biología Tropical, México, 45 (3): 1075-1083.

Chagas Soares, F.das. 1985. Seletividade em redes de emalhar utilizadas na captura de camarões «rosa» Penaeus brasiliensis Latreille, 1817 e Penaeus paulensis Pèrez-Farfante, 1967, na região lagunar-estuarina de Cananéia, São Paulo. Boletim do Instituto de Pesca, São Paulo, 12 (2): 123-142.

Chapman, D.W. 1968. Production, p. 182-196. In: W.E. Ricker (Ed.). Methods for Assessment of Fish Production in Fresh Waters. Oxford, Blackwell Scientific Publications, 313p.

Cook, H.L. 1966. A generic key to the protozoean, mysis and postlarval stages of the littoral Penaeidae of the north western Gulf of Mexico. Fishery Bulletin, London, 65: 437-447. 
D'INCAO, F. 1984. Estudo sobre o crescimento de Penaeus (Farfantepenaeus) paulensis Perez-Farfante, 1967, da lagoa dos Patos, RS. Atlântica, Rio Grande, 7: 73-84.

1991. Pesca e biologia de Penaeus paulensis na lagoa dos Patos, RS, Brasil. Atlântica, Rio Grande, 13 (1): 159169.

D'incao, F \& D. Calazans. 1978. Relações biométricas do “camarão rosa" Penaeus paulensis Perez-Farfante, 1967, na lagoa dos Patos, RS. Atlântica, Rio Grande, 3: 57-66.

Du Preez, H.H. \& A. Mclachlan. 1984. Biology of the three-spot swimming crab Ovalipes punctatus (de Haan). I. Morphometrics and relative growth (Decapoda, Portunidae). Crustaceana, Leiden, 47: 72-82

Esteves, F.A. 1998. Lagoa Imboassica: impactos antrópicos, propostas mitigadoras e sua importância para a pesquisa ecológica, p. 402-429. In: F.A. Esteves (Ed.). Ecologia das Lagoas Costeiras do Parque Nacional da Restinga de Jurubatiba e do Município de Macaé (RJ). Rio de Janeiro, NUPEM/ UFRJ, 464p.

Faria, B.M.; M.S. Suzuki,; M.M. Petrucio \& A.E. Prast. 1998. Changes in the metabolism of a Brazilian lagoon related to man-made marine entrances. Internationale Verenigung für Theoretishe und Angewandte Limnologie, Stuttgard, 26: $1442-1444$.

Fernandes, V.O. 1998. Variação temporal e espacial na composição da comunidade perifítica na lagoa Imboassica, p. 221236. In: F.A. Esteves (Ed.). Ecologia das Lagoas Costeiras do Parque Nacional da Restinga de Jurubatiba e do Município de Macaé (RJ). Rio de Janeiro, NUPEM/UFRJ, 464p.

Frota, L.O.R. \& E.P. Caramaschi. 1998. Aberturas artificiais da barra da lagoa Imboassica e seus efeitos sobre a fauna de peixes, p. 327-350. In: F.A. Esteves (Ed.). Ecologia das Lagoas Costeiras do Parque Nacional da Restinga de Jurubatiba e do Município de Macaé (RJ). Rio de Janeiro, NUPEM/ UFRJ, 464p.

Griffith, D.R.W. \& J.M. Wigglesworth. 1993. Growth rhytms in the shrimp Penaeus vannamei and P. schmitti. Marine Biology, Berlim, 115: 295-299.

Haywood, M.D.E. \& D.J. Staples. 1993. Field estimates of growth and mortality of juvenile banana prawns (Penaeus merguiensis). Marine Biology, Berlin, 116: 407-416.

Kauffman, K. W. 1981. Fitting and using growth curves. Oecologia, Paris, 49: 293-299.

LARES, L.B. \& N.A. KHANDKER. 1976. Length-weight relations of pink spotted shrimp Penaeus brasiliensis Latreille of Caribbean sea. Boletín del Instituto Oceanografico de la Universidad de Oriente, México, 15 (2): 199-200.

LE CREN, E.D. 1951. The length-weight relationship and seasonal cycle in gonad weight and condition in the perch (Perca fluviatilis). Journal of Animal Ecology, London, 20: 201219.

MelLo, J. DE T.C. 1973. Estudo populacional do camarão "rosa", Penaeus brasiliensis (Latreille,1817) e Penaeus paulensis (PèrezFarfante, 1967). Boletim do Instituto de Pesca, São Paulo, 2 (2): 19-65.

Mohammed, H.M.A.; J.M. Bishop \& X. Xu. 1996. Population characteristics of green tiger prawns, Penaeus semisulcatus, in Kuwait waters prior to the Gulf War. Hydrobiologia, Dordrecht, 337: 37-47.
Palacios, J.A.; J.A. Rodríguez \& R.A. Angulo. 1993. Estructura poblacional de Penaeus stylirostris (Decapoda: Penaeidae), en el Golfo de Nicoya, Costa Rica. Revista de Biología Tropical, San José, 41 (2): 233-237.

Palma-Silva, C. 1998. Crescimento e produção de Typha domingensis Pers. na lagoa Imboassica, p. 205-220. In: F.A. Esteves (Ed.). Ecologia das Lagoas Costeiras do Parque Nacional da Restinga de Jurubatiba e do Município de Macaé (RJ). Rio de Janeiro, NUPEM/UFRJ, 464p.

Palma-Silva, C.; E.F. Albertoni \& F.A. Esteves. 2000. Eleocharis mutata (L.) Roem. Et Schult. subject to drawdowns in a tropical coastal lagoon, State of Rio de Janeiro, Brazil. Plant Ecology, Dordrecht, 148: 157-164.

Panosso, R.F.; J.L. Attayde, \& D. Muehe. 1998. Morfometria das lagoas Imboassica, Cabiúnas, Comprida e Carapebus: Implicações para seu funcionamento e manejo, p. 91-108. In: F.A. Esteves (Ed.). Ecologia das Lagoas Costeiras do Parque Nacional da Restinga de Jurubatiba e do Município de Macaé (RJ). Rio de Janeiro, NUPEM/UFRJ, 464p.

Pérez-Farfante, I. 1969. Western atlantic shrimps of the genus Penaeus. Fishery Bulletin, London, 67 (3): 461-591.

. 1970. Diagnostic characteres of juveniles of the shrimps Penaeus aztecus aztecus, $P$. duorarum duorarum and P. brasiliensis (Crustacea, Decapoda, Penaeidae). US Fish and Wildlife Service, London, Special Scientific Report Fisheries, n. 599.

1988. Illustrated key to Penaeoid shrimps of commerce in the Americas. US Department of Commerce, NOAA Technical Report NMFS 64.

Pérez-Farfante, I. \& B. Kensley. 1997. Penaeoid and Sergestoid shrimps and prawns of the world - Keys and diagnosis for the families and genera. Mémoires du Muséum National D'Histoire Naturelle, Paris, 175: 1-233.

Petrucio, M.M. 1998. Caracterização das Lagoas Imboassica, Cabiúnas, Comprida e Carapebus a partir da Temperatura, Salinidade, Condutividade, Alcalinidade, $\mathrm{O}_{2}$ Dissolvido, $\mathrm{pH}$, Transparência e Material em Suspensão, p. 109-121. In: F.A.Esteves (Ed.). Ecologia das Lagoas Costeiras do Parque Nacional da Restinga de Jurubatiba e do Município de Macaé (RJ). Rio de Janeiro, NUPEM/UFRJ, 464p.

Primavera, J.H. 1998. Mangroves as nurseries: shrimp populations in mangrove and non-mangrove habitats. Estuarine Coastal and Shelf Science, London, 46: 457-464.

RADAMBRASIL. 1983. Levantamento de Recursos Naturais. Rio de Janeiro, Ministério das Minas e Energia, vol. 32, 775p.

SOKAL, R.R. \& F.J. RoHLF. 1979. Biometria - Principios y métodos estadísticos en la investigación biológica. Madrid, $\mathrm{H}$. Blume Ediciones, 832p.

Staples, D.J. \& D.S. Heales. 1991. Temperature and salinity optima for growth and survival of juvenile banana prawns Penaeus merguiensis. Journal of Experimental Marine Biology and Ecology, Amsterdam, 154: 251-274.

Subramaniam, S.P. 1990. Chwaka Bay (Zanzibar, East Africa) as a nursery ground for penaeid prawns. Hydrobiologia, Dordrecht, 208: 111-1222.

Tesh, F.W. 1968. Age and Growth, p. 93-123. In: W.E. Ricker (Ed.). Methods for Assessment of Fish Production in Fresh Waters. Oxford, Blackwell Scientific Publications, 313p. Valentini, H.; F. D'incao; L.F. Rodrigues; J.E. Rebelo Neto \& E. 
RAHN. 1991. Análise da pesca do camarão-rosa (Penaeus brasiliensis e Penaeus paulensis) nas regiões sudeste e sul do Brasil. Atlântica, Rio Grande, 13 (1): 143-157.

VAzzoler, A.E.A. \& G. VAzzoler. 1965. Relation between condiction factor and sexual development in Sardinella aurita (Cuv. \& Val., 1847). Anais da Academia Brasileira de Ciências, Rio de Janeiro, 37 (supl.): 353-359.

Villela, M.J.; P.A.S. Costa \& J.L.Valentin. 1997. Crescimento e mortalidade de juvenis do camarão rosa (Penaeus brasiliensis Latreille, 1817) na lagoa de Araruama, Rio de Janeiro. Revista Brasileira de Biologia, Rio de Janeiro, 57 (3): 487-499.
WATSON, R.A. \& C.T. TuRnbull. 1993. Migration and growth of two tropical penaeid shrimps within Torres Strait, northern Australia. Fisheries Research, London, 17: 353-368.

Weatherley, A.H. 1972. Growth and Ecology of Fish Populations. London, Academic Press, 293p.

Weatherley, A.H. \& H.S. Gill. 1987. The Biology of Fish Growth. London, Academic Press, 443p.

Zenkner, H.H. \& J.L. Agnes. 1977. Distribuição do camarão rosa Penaeus brasiliensis e Penaeus paulensis ao longo da costa Sudeste e Sul do Brasil. SUDEPE, Série Documentos Técnicos, São Paulo, vol. 21, 105p.

Recebido em 09.VII.2002; aceito em 13.VIII.2003.

Revista Brasileira de Zoologia 20 (3): 409-418, setembro 2003 